\title{
ASYMPTOTIC BEHAVIOR OF STRONGLY DAMPED NONLINEAR BEAM EQUATIONS
}

\author{
AHMED Y. ABDALLAH
}

\begin{abstract}
For $\xi \in[1 / 2,3 / 4]$, the existence of the global attractor for the evolutionary equation corresponding to the following strongly damped nonlinear beam equation $\left(1+\beta A^{1 / 2}\right) u_{t t}+\delta A^{1 / 2} u_{t}+\alpha A u+g\left(\|u\|_{\xi-1 / 4}^{2}\right) A^{1 / 2} u=f$, $t>0$, has been studied in $\mathcal{D}_{H}\left(A^{\xi}\right) \times \mathcal{D}_{H}\left(A^{\xi-1 / 4}\right)$. Such an equation is related to a nonlinear beam equation as well as Timoshenko's equation.

The main difficulty of our work comes from the terms $\beta A^{1 / 2} u_{t t}$ and $g\left(\|u\|_{\xi-1 / 4}^{2}\right) A^{1 / 2} u$, representing the rotational inertia of the beam and the tension within the beam due to its extensibility, respectively. We overcome the difficulty of introducing the solution, bounded absorbing set, and $\kappa$ contracting property by carefully using the fractional power theory and suitable time-uniform a priori estimates.
\end{abstract}

1. Introduction. The asymptotic behavior and global dynamics for nonlinear beam equations of the following form

$$
u_{t t}+\delta u_{t}+\alpha u_{x x x x}+\left(a_{0}+a_{1} \int_{0}^{1}\left|u_{x}\right|^{2} d x\right) u_{x x}=f, \quad t>0,
$$

where $a_{0} \in \mathbb{R}$ and $\delta, \alpha, a_{1}>0$, have been carefully studied by many researchers, cf., $[4,8, \mathbf{1 3}, \mathbf{1 4}]$. The asymptotic behavior for different types of nonlinear beam equations has been extensively studied in the literature $[2,3,5,6,7,9,12]$.

In $[\mathbf{1}, \mathbf{1 1}, \mathbf{1 5}]$ and the references therein, Timoshenko's equation

$$
u_{t t}+\beta u_{x x t t}+\alpha u_{x x x x}+\left(\int_{0}^{1}\left|u_{x}\right|^{2} d x\right) u_{x x}=f, \quad t>0,
$$

has been studied, where $\alpha, \beta>0$.

2010 AMS Mathematics subject classification. Primary 35B41, 35B45.

Keywords and phrases. Beam equation, bounded dissipative, $\kappa$-contracting, global attractor.

Received by the editors on September 30, 2014. 
Here, we introduce a nonlinear equation related to equations (1.1) and (1.2). Indeed, consider the Hilbert space $H=L^{2}(0,1)$ whose inner product and norm are denoted by $\langle\cdot, \cdot\rangle_{0}$ and $\|\cdot\|_{0}$, respectively. For a given $\xi \in[1 / 2,3 / 4]$, we shall study the existence of the global attractor for an abstract evolutionary equation corresponding to the following nonlinear strongly damped beam equation:

$$
\left(1+\beta A^{1 / 2}\right) u_{t t}+\delta A^{1 / 2} u_{t}+\alpha A u+g\left(\|u\|_{\xi-1 / 4}^{2}\right) A^{1 / 2} u=f, \quad t>0
$$

with initial conditions

$$
u(0)=u_{0} \quad \text { and } \quad u_{t}(0)=u_{1},
$$

where the closed linear operator $A$, the functions $f$ and $g$, and the parameters $\alpha, \beta$, and $\delta$, are given by assumptions $(A 1)-(A 4)$ below.

Taking into account assumption $(A 4)$ and the theory of fractional powers discussed below, it is clear that, for $\beta=0, \xi=1 / 2$, damping $\delta u_{t}$ and $g(u)=a_{0}+a_{1}\left\|A^{1 / 4} u\right\|_{0}^{2}$, equation (1.3) becomes identical to equation (1.1), and for $\xi=1 / 2, \delta=0$ and $g(u)=\left\|A^{1 / 4} u\right\|_{0}^{2}$, equation (1.3) becomes identical to equation (1.2). From the definition of the closed linear operator $A$ given by assumption $(A 4)$ below, it is clear that the hinged boundary conditions are considered in this work.

The nonlinear beam equation (1.3) can be considered as a mathematical model for small transversal vibrations of an extensible beam, where $u(x, t)$ is the transverse deflection of the beam, $\delta$ and $\beta$ are the parameters related to the structural strong damping and the rotational inertia of the beam, respectively. The nonlinear term in the equation is the tension within the beam due to its extensibility, and the function $f$ stands for an external input.

Within this work, we assume the following:

(A1) $\alpha, \delta, \beta>0$ and $\xi \in[1 / 2,3 / 4]$.

(A2) $f \in H=L^{2}(0,1)$, where the inner product and norm in $H$ are denoted by $\langle\cdot, \cdot\rangle_{0}$ and $\|\cdot\|_{0}$, respectively.

$(A 3) g:[0, \infty) \rightarrow \mathbb{R}$ is locally Lipschitz continuous and there exist $r, K_{0}, K_{1} \geq 0$, with $K_{1}<\pi^{2} \alpha$, such that

$$
\begin{gathered}
\int_{r}^{s} g(y) d y \geq-\left(K_{0}+K_{1} s\right), \quad g\left(s_{1}\right) \geq g\left(s_{2}\right), \\
\text { for all } s \geq r, \quad s_{1} \geq s_{2} \geq r .
\end{gathered}
$$


Choosing

$$
K_{2}=K_{2}(r)=\frac{3}{2} r \max _{y \in[0, r]}|g(y)|
$$

it is clear that, for $0 \leq s<r$,

$$
\begin{gathered}
\int_{0}^{s} g(y) d y \geq-\int_{0}^{s}|g(y)| d y \geq-r \max _{y \in[0, r]}|g(y)| \geq-K_{2}, \\
\frac{1}{2} \int_{0}^{s} g(y) d y-s g(s) \leq \frac{1}{2} \int_{0}^{s}|g(y)| d y+s|g(s)| \leq \frac{3}{2} r \max _{y \in[0, r]}|g(y)|=K_{2},
\end{gathered}
$$

and, for $s \geq r$,

$$
\begin{aligned}
\int_{0}^{s} g(y) d y= & \int_{0}^{r} g(y) d y+\int_{r}^{s} g(y) d y \geq-r \max _{y \in[0, r]}|g(y)| \\
& +\int_{r}^{s} g(y) d y \geq-\left(K_{2}+K_{0}+K_{1} s\right), \\
\frac{1}{2} \int_{0}^{s} g(y) d y-s g(s) & =\frac{1}{2} \int_{0}^{r} g(y) d y+\frac{1}{2} \int_{r}^{s} g(y) d y-(s-r) g(s)-r g(s) \\
& =\frac{1}{2} \int_{0}^{r} g(y) d y+\int_{r}^{s}\left(\frac{1}{2} g(y)-g(s)\right) d y-r g(s) \\
& \leq \frac{1}{2} r \max _{y \in[0, r]}|g(y)|-\frac{1}{2} \int_{r}^{s} g(y) d y-r g(r) \\
& \leq \frac{1}{2}\left(K_{0}+K_{1} s\right)+K_{2} .
\end{aligned}
$$

Hence, for $s \geq 0$, we have

$$
\begin{gathered}
\frac{1}{2} \int_{0}^{s} g(y) d y-s g(s) \leq \frac{1}{2}\left(K_{0}+K_{1} s\right)+K_{2}, \\
\int_{0}^{s} g(y) d y \geq-\left(K_{2}+K_{0}+K_{1} s\right) .
\end{gathered}
$$

(A4) $A: \mathcal{D}_{H}(A) \subset H \rightarrow H$ is the closed linear operator defined as follows

$$
A \varphi=\varphi_{x x x x}, \quad \text { for all } \varphi \in \mathcal{D}_{H}(A),
$$

where the domain of $A$ in $H$ is given by

$$
\mathcal{D}_{H}(A)=\left\{\varphi \in H^{4}(0,1): \varphi(0)=\varphi_{x x}(0)=\varphi(1)=\varphi_{x x}(1)=0\right\} .
$$


Following [13], it can be shown that $A$ is a densely defined, selfadjoint, positive definite operator with compact resolvent $A^{-1}$. The spectrum of $A$ consists of the eigenvalues $\lambda_{n}=(n \pi)^{4}, n \in \mathbb{N}$, with eigenvectors $e_{n}=2^{1 / 2} \sin (n \pi x), n \in \mathbb{N}$.

Along the lines of the fractional power theory $[\mathbf{1 0}, \mathbf{1 3}]$, for $\xi \geq 0$, $V^{\xi}=\mathcal{D}_{H}\left(A^{\xi}\right)$ is a Hilbert space where $V^{0}=H$, whose inner product and norm are given by

$$
\langle\varphi, \psi\rangle_{\xi}=\left\langle A^{\xi} \varphi, A^{\xi} \psi\right\rangle_{0}, \quad\|\varphi\|_{\xi}=\left\langle A^{\xi} \varphi, A^{\xi} \varphi\right\rangle_{0}^{1 / 2}=\left\|A^{\xi} \varphi\right\|_{0},
$$

for all $\varphi, \psi \in V^{\xi}$. If $\xi>\eta \geq 0$, then $V^{\xi}$ is compactly embedded in $V^{\eta}$ and

$$
\|\varphi\|_{\eta} \leq \pi^{4(\eta-\xi)}\|\varphi\|_{\xi} \leq\|\varphi\|_{\xi}, \quad \text { for all } \varphi \in V^{\xi} .
$$

For $\xi, \eta \geq 0$, we have

$$
\begin{aligned}
A^{\xi} A^{\eta} & =A^{\eta} A^{\xi}=A^{\xi+\eta} \quad \text { on } V^{\xi+\eta}, \\
\left\langle A^{\xi} \varphi, \psi\right\rangle_{0} & =\left\langle\varphi, A^{\xi} \psi\right\rangle_{0}, \quad \text { for all } \varphi, \psi \in V^{\xi} .
\end{aligned}
$$

Considering the linear operator $A: \mathcal{D}_{V \xi}(A) \subset V^{\xi} \rightarrow V^{\xi}, \xi \geq 0$, where the domain of $A$ in $V^{\xi}$ is given by $\mathcal{D}_{V^{\xi}}(A)=V^{\xi+1}$, one can show that $A$ is a sectorial operator and $-A$ generates an analytic semigroup on $V^{\xi}$. Moreover, we have

$$
\begin{gathered}
A^{1 / 2} \varphi=-\varphi_{x x}, \quad \text { for all } \varphi \in V^{1 / 2} \\
\|\varphi\|_{\xi}=\left\|\frac{\partial^{4 \xi} \varphi}{\partial x^{4 \xi}}\right\|_{0}, \quad \text { for all } \varphi \in V^{\xi}, 4 \xi=0,1,2, \ldots
\end{gathered}
$$

For $\xi, \eta \geq 0$, we shall consider the Hilbert space $E_{\xi, \eta}=V^{\xi} \times V^{\eta}$, whose inner product and norm are given by:

$$
\begin{gathered}
\left\langle\Psi_{0}, \Psi_{1}\right\rangle_{E_{\xi, \eta}}=\left\langle\varphi_{0}, \varphi_{1}\right\rangle_{\xi}+\left\langle\psi_{0}, \psi_{1}\right\rangle_{\eta}, \\
\left\|\Psi_{0}\right\|_{E_{\xi, \eta}}=\left\langle\Psi_{0}, \Psi_{0}\right\rangle_{E_{\xi, \eta}}^{1 / 2}
\end{gathered}
$$

for all $\Psi_{i}=\left(\varphi_{i}, \psi_{i}\right)^{T} \in E_{\xi, \eta}, i=0,1$.

The objective of this research is to prove the existence of the global attractor for an abstract dynamical system corresponding to the initial value problem (1.3)-(1.4) in $E_{\xi, \xi-1 / 4}$. The existence of a global attractor for the solution semiflow of a given dynamical system is very 
important because it is the depository of all the longtime dynamics of such a system. The background material in regard to the theory of global attractors can be found in $[\mathbf{8}, \mathbf{1 3}]$.

Fixing $\xi \in[1 / 2,3 / 4]$, this work is organized as follows. In Section 2, the initial value problem (1.3)-(1.4) will be represented as an abstract evolutionary equation in $E_{\xi, \xi-1 / 4}$ and the standard semigroup theory will be used to prove the existence of a local solution for this abstract equation. In Section 3, we shall prove that the solution exists globally and the semiflow generated by these solutions is bounded dissipative. In Section 4, we shall show that the solution semiflow is $\kappa$-contracting. Using these results, along the lines of the classical theory of global attractors, it follows that the solution semiflow possesses a global attractor.

The difficulty of studying the existence of the global attractor for the evolutionary equation related to the initial value problem (1.3)-(1.4) comes from the terms:

$$
\beta A^{1 / 2} u_{t t} \quad \text { and } \quad g\left(\|u\|_{\xi-1 / 4}^{2}\right) A^{1 / 2} u
$$

representing the rotational inertia of the beam and the tension within the beam due to its extensibility, respectively. Carefully using the fractional power theory and suitable time-uniform a priori estimates, we overcome the difficulty of presenting the solution semiflow, bounded absorbing set, and $\kappa$-contracting property for the solution semiflow.

2. Formulation and well posedness. Letting $v=u_{t}$, we can reformulate (1.3)-(1.4) as the following first-order evolution equation

$$
\begin{gathered}
B \Phi_{t}=C \Phi+F_{\xi}(\Phi), \quad t>0 \\
\Phi(0)=\Phi_{0}=\left(u_{0}, u_{1}\right)^{T},
\end{gathered}
$$

where $\Phi=(u, v)^{T}$,

$$
\begin{gathered}
B=\left(\begin{array}{cc}
I & 0 \\
0 & I+\beta A^{1 / 2}
\end{array}\right), \quad C=\left(\begin{array}{cc}
0 & I \\
-\alpha A & -\delta A^{1 / 2}
\end{array}\right), \\
F_{\xi}(\Phi)=\left(0, J_{\xi}(u)\right)^{T} \text { and } \quad J_{\xi}(u)=f-g\left(\|u\|_{\xi-1 / 4}^{2}\right) A^{1 / 2} u .
\end{gathered}
$$


Using (1.7) and the assumption that $g$ is locally Lipschitz continuous, it follows that, for $K>0, \varphi, \psi \in V^{\xi}$ with $\xi \geq 1 / 4$,

$$
\|\varphi\|_{\xi-1 / 4} \leq K, \quad\|\psi\|_{\xi-1 / 4} \leq K
$$

there exist constants $k_{i}=k_{i}(K)>0, i=0,1$, such that

$$
\begin{aligned}
\left|g\left(\|\varphi\|_{\xi-1 / 4}^{2}\right)-g\left(\|\psi\|_{\xi-1 / 4}^{2}\right)\right| & \leq k_{0}\left|\|\varphi\|_{\xi-1 / 4}^{2}-\|\psi\|_{\xi-1 / 4}^{2}\right| \\
& \leq k_{1}\|\varphi-\psi\|_{\xi} .
\end{aligned}
$$

Lemma 2.1. Given $\xi \in[1 / 2,3 / 4]$, the nonlinear operator

$$
B^{-1} F_{\xi}(\Phi)=\left(0,\left(I+\beta A^{1 / 2}\right)^{-1} J_{\xi}(u)\right)^{T}, \quad \text { for all } \Phi=(u, v)^{T}
$$

is locally Lipschitz continuous in $E_{\xi, \xi-1 / 4}$ and $E_{3 / 4,1 / 2}$.

Proof. Given $\xi \in[1 / 2,3 / 4]$, let $k_{2}>0$ and $\Phi_{i}=\left(u_{i}, v_{i}\right)^{T} \in E_{\xi, \xi-1 / 4}$ be such that

$$
\left\|\Phi_{i}\right\|_{E_{\xi, \xi-1 / 4}} \leq k_{2}, \quad i=0,1 .
$$

Then, from equation (1.7) and the fact that $\left(I+\beta A^{1 / 2}\right)^{-1}$ is a bounded operator from $H$ into $V^{1 / 2}$, we find that there exist a constant $k_{3}>0$ such that

$$
\begin{aligned}
& \left\|B^{-1} F_{\xi}\left(\Phi_{0}\right)-B^{-1} F_{\xi}\left(\Phi_{1}\right)\right\|_{E_{\xi, \xi-1 / 4}} \\
& =\left\|\left(I+\beta A^{1 / 2}\right)^{-1}\left[g\left(\left\|u_{0}\right\|_{\xi-1 / 4}^{2}\right) A^{1 / 2} u_{0}-g\left(\left\|u_{1}\right\|_{\xi-1 / 4}^{2}\right) A^{1 / 2} u_{1}\right]\right\|_{\xi-1 / 4} \\
& \leq\left\|\left(I+\beta A^{1 / 2}\right)^{-1}\left[g\left(\left\|u_{0}\right\|_{\xi-1 / 4}^{2}\right) A^{1 / 2} u_{0}-g\left(\left\|u_{1}\right\|_{\xi-1 / 4}^{2}\right) A^{1 / 2} u_{1}\right]\right\|_{1 / 2} \\
& \leq k_{3}\left\|g\left(\left\|u_{0}\right\|_{\xi-1 / 4}^{2}\right) A^{1 / 2} u_{0}-g\left(\left\|u_{1}\right\|_{\xi-1 / 4}^{2}\right) A^{1 / 2} u_{1}\right\|_{0} \\
& \leq k_{3}\left(\left|g\left(\left\|u_{0}\right\|_{\xi-1 / 4}^{2}\right)\right|\left\|u_{0}-u_{1}\right\|_{1 / 2}+\mid g\left(\left\|u_{0}\right\|_{\xi-1 / 4}^{2}\right)\right. \\
& \left.\quad-g\left(\left\|u_{1}\right\|_{\xi-1 / 4}^{2}\right) \mid\left\|u_{1}\right\|_{1 / 2}\right) .
\end{aligned}
$$

From equations (1.7) and (2.1), along with the fact that $g$ is locally Lipschitz continuous, it follows that there exists a constant $k_{4}=$ $k_{4}\left(k_{2}\right)>0$ such that

$$
\begin{aligned}
& \left\|B^{-1} F_{\xi}\left(\Phi_{0}\right)-B^{-1} F_{\xi}\left(\Phi_{1}\right)\right\|_{E_{\xi, \xi-1 / 4}} \\
& \quad \leq k_{4}\left\|u_{0}-u_{1}\right\|_{\xi} \leq k_{4}\left\|\Phi_{0}-\Phi_{1}\right\|_{E_{\xi, \xi-1 / 4}} .
\end{aligned}
$$

Thus, $B^{-1} F_{\xi}$ is locally Lipschitz continuous in $E_{\xi, \xi-1 / 4}$. Similarly, one can see that $B^{-1} F_{\xi}$ is locally Lipschitz continuous in $E_{3 / 4,1 / 2}$. 
For $\xi \in[1 / 2,3 / 4]$, consider the linear operator

$$
B^{-1} C: \mathcal{D}_{E_{\xi, \xi-1 / 4}}\left(B^{-1} C\right) \subset E_{\xi, \xi-1 / 4} \rightarrow E_{\xi, \xi-1 / 4},
$$

given by

$$
B^{-1} C=\left(\begin{array}{cc}
0 & I \\
-\alpha\left(I+\beta A^{1 / 2}\right)^{-1} A & -\delta\left(I+\beta A^{1 / 2}\right)^{-1} A^{1 / 2}
\end{array}\right),
$$

whose domain in $E_{\xi, \xi-1 / 4}$ is given by $\mathcal{D}_{E_{\xi, \xi-1 / 4}}\left(B^{-1} C\right)=E_{1, \xi}$. It can be shown that $-B^{-1} C$ is a sectorial operator and $B^{-1} C$ is the infinitesimal generator of an analytic contraction semigroup in $E_{\xi, \xi-1 / 4}$. In this case, using Lemma 2.1, and the standard semigroup theory [13], we get the following result.

Lemma 2.2. For $\xi \in[1 / 2,3 / 4]$, consider the initial value problem

$$
\begin{gathered}
\Phi_{t}=B^{-1} C \Phi+B^{-1} F_{\xi}(\Phi), \quad t>0 \\
\Phi(0)=\Phi_{0}=\left(u_{0}, u_{1}\right)^{T} .
\end{gathered}
$$

(a) Given $\Phi_{0}=\left(u_{0}, u_{1}\right)^{T} \in E_{\xi, \xi-1 / 4}$, equation (2.2) has a unique maximal solution

$$
\begin{gathered}
\Phi\left(t ; \xi, \Phi_{0}\right)=\left(u(t), u_{t}(t)\right)^{T}, \\
t \in[0, \tau) \quad \text { and } \quad \tau=\tau\left(\Phi_{0}\right)>0,
\end{gathered}
$$

in $E_{\xi, \xi-1 / 4}$ satisfying $(2.2)$ for $t \in[0, \tau)$, such that

$$
\begin{aligned}
\left(u(\cdot), u_{t}(\cdot)\right)^{T} & =C\left([0, \tau), E_{\xi, \xi-1 / 4}\right) \\
& \cap C^{1}\left((0, \tau), E_{\xi, \xi-1 / 4}\right) \cap C\left((0, \tau), E_{1, \xi}\right),
\end{aligned}
$$

where $E_{1, \xi}$ is $\mathcal{D}_{E_{\xi, \xi-1 / 4}}\left(B^{-1} C\right)$. Moreover, if $\tau<\infty$, then

$$
\lim _{t \rightarrow \tau^{-}}\left\|\left(u(t), u_{t}(t)\right)^{T}\right\|_{E_{\xi, \xi-1 / 4}}=\infty .
$$

(b) Given $\Phi_{0}=\left(u_{0}, u_{1}\right)^{T} \in E_{3 / 4,1 / 2}$, equation (2.2) has a unique maximal solution

$$
\begin{gathered}
\Phi\left(t ; \xi, \Phi_{0}\right)=\left(u(t), u_{t}(t)\right)^{T}, \\
t \in[0, \tau) \quad \text { and } \quad \tau=\tau\left(\Phi_{0}\right)>0,
\end{gathered}
$$


in $E_{3 / 4,1 / 2}$ satisfying (2.2) for $t \in[0, \tau)$ such that

$$
\begin{aligned}
\left(u(\cdot), u_{t}(\cdot)\right)^{T} & =C\left([0, \tau), E_{3 / 4,1 / 2}\right) \\
& \cap C^{1}\left((0, \tau), E_{3 / 4,1 / 2}\right) \cap C\left((0, \tau), E_{1,3 / 4}\right),
\end{aligned}
$$

where $E_{1,3 / 4}$ is $\mathcal{D}_{E_{3 / 4,1 / 2}}\left(B^{-1} C\right)$. Moreover, if $\tau<\infty$, then

$$
\lim _{t \rightarrow \tau^{-}}\left\|\left(u(t), u_{t}(t)\right)^{T}\right\|_{E_{3 / 4,1 / 2}}=\infty .
$$

3. Global solutions and dissipativity. Before introducing Lemmas 3.2 and 4.1, we need the following remark to explain the reason for choosing the initial data $\Phi_{0}$ in $E_{3 / 4,1 / 2}$ while studying in $E_{\xi, \xi-1 / 4}$ the global existence of solutions bounded dissipativity, and uniform $\kappa$-contracting property for the solution semiflow associated with the initial value problem $(2.2)$.

Remark 3.1. In both lemmas, we need (1.3) to be satisfied as a first step to complete the proof. Reading (2.3) and (2.5) carefully, one can see that, for $\Phi_{0} \in E_{\xi, \xi-1 / 4}$, the solution given by (2.3) satisfies (2.2), but it does not satisfy (1.3). For $\Phi_{0} \in E_{3 / 4,1 / 2}$, the solution given by (2.5) satisfies (2.2), and it consequently satisfies (1.3).

The key idea for proving the following lemma is applying suitable time-uniform a priori estimates.

Lemma 3.2. In $E_{\xi, \xi-1 / 4}, \xi \in[1 / 2,3 / 4]$, for each $\Phi_{0} \in E_{\xi, \xi-1 / 4}$, the unique solution of the initial value problem (2.2) given by equation (2.3), exists globally and the associated semiflow $S_{\xi}(t)$ generated by these solutions is bounded dissipative.

Proof. Let

$$
\xi \in[1 / 2,3 / 4], \quad \Phi_{0} \in E_{3 / 4,1 / 2}
$$

and

$$
S_{\xi}(t) \Phi_{0}=\Phi(t)=\left(u(t), u_{t}(t)\right)^{T}
$$

be the solution of equation (2.2) in $E_{3 / 4,1 / 2}$, given by equation (2.5). Recalling Remark 3.1, it is clear that equation (1.3) is satisfied for 
$t \in(0, \tau)$. For $\varepsilon>0$, if we consider the inner product of equation (1.3) with $A^{2 \xi-1}\left(u_{t}+\varepsilon u\right)$ in $H$, taking into account equation (1.8), we obtain

$$
\frac{d}{d t} \Theta_{\xi}(t)+\Pi_{\xi}(t)=0, \quad \text { for all } t \in(0, \tau)
$$

where

$$
\begin{aligned}
\Theta_{\xi}(t)= & \frac{1}{2}\left\|u_{t}\right\|_{\xi-1 / 2}^{2}+\frac{\beta}{2}\left\|u_{t}\right\|_{\xi-1 / 4}^{2}+\frac{\alpha}{2}\|u\|_{\xi}^{2}+\frac{\varepsilon \delta}{2}\|u\|_{\xi-1 / 4}^{2} \\
& -\left\langle f, A^{2 \xi-1} u\right\rangle_{0}+\frac{1}{2} \int_{0}^{\|u\|_{\xi-1 / 4}^{2}} g(y) d y \\
& +\varepsilon\left\langle u_{t}, u\right\rangle_{\xi-1 / 2}+\varepsilon \beta\left\langle u_{t}, u\right\rangle_{\xi-1 / 4}
\end{aligned}
$$

and

$$
\begin{aligned}
\Pi_{\xi}(t)= & (\delta-\varepsilon \beta)\left\|u_{t}\right\|_{\xi-1 / 4}^{2}-\varepsilon\left\|u_{t}\right\|_{\xi-1 / 2}^{2}+\varepsilon \alpha\|u\|_{\xi}^{2} \\
& +\varepsilon g\left(\|u\|_{\xi-1 / 4}^{2}\right)\|u\|_{\xi-1 / 4}^{2}-\varepsilon\left\langle f, A^{2 \xi-1} u\right\rangle_{0} .
\end{aligned}
$$

From Young's inequality and (1.7), we find

$$
\begin{gathered}
\left|\left\langle u_{t}, u\right\rangle_{\xi-1 / 2}\right| \leq \frac{1}{2}\left\|u_{t}\right\|_{\xi-1 / 2}^{2}+\frac{1}{2}\|u\|_{\xi-1 / 2}^{2} \leq \frac{1}{2}\left\|u_{t}\right\|_{\xi-1 / 4}^{2}+\frac{1}{2}\|u\|_{\xi}^{2}, \\
\left|\left\langle u_{t}, u\right\rangle_{\xi-1 / 4}\right| \leq \frac{1}{2}\left\|u_{t}\right\|_{\xi-1 / 4}^{2}+\frac{1}{2}\|u\|_{\xi}^{2} \\
\left|\left\langle f, A^{2 \xi-1} u\right\rangle_{0}\right| \leq\|f\|_{0}\|u\|_{2 \xi-1} \leq \frac{\|f\|_{0}^{2}}{2 \varepsilon}+\frac{\varepsilon}{2}\|u\|_{\xi}^{2} .
\end{gathered}
$$

Using (3.2) and (3.4), we get

$$
\begin{aligned}
\Theta_{\xi}(0) \leq & \frac{1}{2}\left\|u_{t}(0)\right\|_{\xi-1 / 2}^{2}+\frac{\beta}{2}\left\|u_{t}(0)\right\|_{\xi-1 / 4}^{2}+\frac{\alpha}{2}\|u(0)\|_{\xi}^{2} \\
& +\frac{\varepsilon}{2} \delta\|u(0)\|_{\xi-1 / 4}^{2}+\frac{\|f\|_{0}^{2}}{2 \varepsilon}+\frac{\varepsilon}{2}\|u(0)\|_{\xi}^{2}+\frac{1}{2} \int_{0}^{\|u(0)\|_{\xi-1 / 4}^{2}} \\
& \cdot g(y) d y+\frac{\varepsilon}{2}(1+\beta)\left(\left\|u_{t}(0)\right\|_{\xi-1 / 4}^{2}+\|u(0)\|_{\xi}^{2}\right) .
\end{aligned}
$$


From (1.7), it follows that

$$
\begin{aligned}
\Theta_{\xi}(0) \leq & \frac{1}{2}(1+\beta+\varepsilon(1+\beta))\left\|u_{t}(0)\right\|_{\xi-1 / 4}^{2}+\frac{\|f\|_{0}^{2}}{2 \varepsilon} \\
& +\frac{1}{2}\left(\alpha+\varepsilon(2+\delta+\beta)+\max _{y \in\left[0,\|u(0)\|_{\xi}^{2}\right]}|g(y)|\right)\|u(0)\|_{\xi}^{2} .
\end{aligned}
$$

Again, recalling (3.2) and (3.4), we have

$$
\begin{gathered}
\frac{1}{2}\left\|u_{t}(t)\right\|_{\xi-1 / 2}^{2}+\frac{\beta}{2}\left\|u_{t}(t)\right\|_{\xi-1 / 4}^{2}+\frac{\alpha}{2}\|u(t)\|_{\xi}^{2}+\frac{\varepsilon \delta}{2}\|u(t)\|_{\xi-1 / 4}^{2} \\
-\frac{\|f\|_{0}^{2}}{2 \varepsilon}-\frac{\varepsilon}{2}\|u(t)\|_{\xi}^{2}+\frac{1}{2} \int_{0}^{\|u(t)\|_{\xi-1 / 4}^{2}} g(y) d y \\
-\frac{1}{2} \varepsilon(1+\beta)\left(\left\|u_{t}(t)\right\|_{\xi-1 / 4}^{2}+\|u(t)\|_{\xi}^{2}\right) \leq \Theta_{\xi}(t), \\
\frac{1}{2}(\beta-\varepsilon(1+\beta))\left\|u_{t}(t)\right\|_{\xi-1 / 4}^{2}+\frac{1}{2}(\alpha-\varepsilon(2+\beta))\|u(t)\|_{\xi}^{2}+\frac{1}{2} \int_{0}^{\|u(t)\|_{\xi-1 / 4}^{2}} g(y) d y \\
\leq \Theta_{\xi}(t)+\frac{\|f\|_{0}^{2}}{2 \varepsilon},
\end{gathered}
$$

From (1.6) and (1.7) we obtain

$$
\begin{aligned}
& \frac{1}{2}(\beta-\varepsilon(1+\beta))\left\|u_{t}(t)\right\|_{\xi-1 / 4}^{2}+\frac{1}{2}\left(\alpha-\pi^{-2} K_{1}-\varepsilon(2+\beta)\right)\|u(t)\|_{\xi}^{2} \\
& \leq \Theta_{\xi}(t)+\frac{\|f\|_{0}^{2}}{2 \varepsilon}+\frac{K_{0}}{2}+\frac{K_{2}}{2} .
\end{aligned}
$$

Taking into account (3.2) and (3.3), we find

$$
\begin{aligned}
\varepsilon \Theta_{\xi}(t)-\Pi_{\xi}(t)= & \frac{3 \varepsilon}{2}\left\|u_{t}\right\|_{\xi-1 / 2}^{2}+\left(\frac{3 \varepsilon \beta}{2}-\delta\right)\left\|u_{t}\right\|_{\xi-1 / 4}^{2}-\frac{\varepsilon \alpha}{2}\|u\|_{\xi}^{2} \\
& +\frac{\varepsilon^{2} \delta}{2}\|u\|_{\xi-1 / 4}^{2}+\frac{\varepsilon}{2} \int_{0}^{\|u\|_{\xi-1 / 4}^{2}} g(y) d y \\
& -\varepsilon g\left(\|u\|_{\xi-1 / 4}^{2}\right)\|u\|_{\xi-1 / 4}^{2} \\
& +\varepsilon^{2}\left\langle u_{t}, u\right\rangle_{\xi-1 / 2}+\varepsilon^{2} \beta\left\langle u_{t}, u\right\rangle_{\xi-1 / 4},
\end{aligned}
$$


using (3.4) it follows that

$$
\begin{aligned}
\varepsilon \Theta_{\xi}(t)-\Pi_{\xi}(t)= & \frac{3 \varepsilon}{2}\left\|u_{t}\right\|_{\xi-1 / 2}^{2}+\left(\frac{3 \varepsilon \beta}{2}-\delta\right)\left\|u_{t}\right\|_{\xi-1 / 4}^{2}-\frac{\varepsilon \alpha}{2}\|u\|_{\xi}^{2} \\
& +\frac{\varepsilon^{2} \delta}{2}\|u\|_{\xi-1 / 4}^{2}+\frac{\varepsilon}{2} \int_{0}^{\|u\|_{\xi-1 / 4}^{2}} g(y) d y \\
& -\varepsilon g\left(\|u\|_{\xi-1 / 4}^{2}\right)\|u\|_{\xi-1 / 4}^{2} \\
& +\frac{1}{2} \varepsilon^{2}(1+\beta)\left(\left\|u_{t}\right\|_{\xi-1 / 4}^{2}+\|u\|_{\xi}^{2}\right) .
\end{aligned}
$$

From (1.6) and (1.7), we get

$$
\begin{aligned}
\varepsilon \Theta_{\xi}(t)-\Pi_{\xi}(t) \leq & \left(\frac{\varepsilon}{2}(1+\beta)(\varepsilon+3)-\delta\right)\left\|u_{t}(t)\right\|_{\xi-1 / 4}^{2} \\
& +\frac{\varepsilon}{2}\left[\varepsilon(\delta+1+\beta)+\pi^{-2} K_{1}-\alpha\right]\|u(t)\|_{\xi}^{2} \\
& +\varepsilon\left(\frac{K_{0}}{2}+K_{2}\right) .
\end{aligned}
$$

In (A3), we assumed that $K_{1}<\pi^{2} \alpha$. For sufficiently small $\varepsilon>0$, inequality (3.7) becomes

$$
\varepsilon \Theta_{\xi}(t)-\Pi_{\xi}(t) \leq \varepsilon\left(\frac{K_{0}}{2}+K_{2}\right) .
$$

Using (3.1), we get

$$
\frac{d}{d t} \Theta_{\xi}(t)+\varepsilon \Theta_{\xi}(t) \leq \varepsilon\left(\frac{K_{0}}{2}+K_{2}\right),
$$

that is,

$$
\frac{d}{d t}\left(e^{\varepsilon t} \Theta_{\xi}(t)\right) \leq \varepsilon\left(\frac{K_{0}}{2}+K_{2}\right) e^{\varepsilon t} .
$$

Integrating the above inequality from 0 into $t$, where $t \in(0, \tau)$, we find

$$
\Theta_{\xi}(t) \leq \Theta_{\xi}(0) e^{-\varepsilon t}+\frac{K_{0}}{2}+K_{2}, \quad \text { for all } t \in[0, \tau) .
$$

Again, since $K_{1}<\pi^{2} \alpha$, we can choose $\varepsilon>0$ sufficiently small such that

$$
\min \left\{\beta-\varepsilon(1+\beta), \alpha-\pi^{-2} K_{1}-\varepsilon(2+\beta)\right\}>0
$$


Choosing

$$
\begin{gathered}
k_{7}=\frac{1}{2} \min \left\{\beta-\varepsilon(1+\beta), \alpha-\pi^{-2} K_{1}-\varepsilon(2+\beta)\right\}, \\
k_{8}=\frac{1}{2} \max \left\{\begin{array}{c}
1+\beta+\varepsilon(1+\beta), \\
\alpha+\varepsilon(2+\delta+\beta)+\max _{y \in\left[0,\|u(0)\|_{\xi}^{2}\right]}|g(y)|
\end{array}\right\},
\end{gathered}
$$

and using (3.5) and (3.6), it follows that

$$
\begin{aligned}
\Theta_{\xi}(0) & \leq k_{8}\left(\left\|u_{t}(0)\right\|_{\xi-1 / 4}^{2}+\|u(0)\|_{\xi}^{2}\right)+\frac{\|f\|_{0}^{2}}{2 \varepsilon} \\
& =k_{8}\left\|\Phi_{0}\right\|_{E_{\xi, \xi-1 / 4}}^{2}+\frac{\|f\|_{0}^{2}}{2 \varepsilon}
\end{aligned}
$$

and

$$
\begin{aligned}
k_{7}\left\|S_{\xi}(t) \Phi_{0}\right\|_{E_{\xi, \xi-1 / 4}}^{2} & =k_{7}\left(\left\|u_{t}(t)\right\|_{\xi-1 / 4}^{2}+\|u(t)\|_{\xi}^{2}\right) \\
& \leq \Theta_{\xi}(t)+\frac{\|f\|_{0}^{2}}{2 \varepsilon}+\frac{K_{0}}{2}+\frac{K_{2}}{2} .
\end{aligned}
$$

In such a case, recalling (3.8) and the previous two inequalities, it follows that for $t \in[0, \tau)$,

$$
\left\|S_{\xi}(t) \Phi_{0}\right\|_{E_{\xi, \xi-1 / 4}}^{2} \leq \frac{1}{k_{7}}\left[\left(k_{8}\left\|\Phi_{0}\right\|_{E_{\xi, \xi-1 / 4}}^{2}+\frac{\|f\|_{0}^{2}}{2 \varepsilon}\right) e^{-\varepsilon t}+k_{9}\right]
$$

where

$$
k_{9}=\frac{\|f\|_{0}^{2}}{2 \varepsilon}+K_{0}+\frac{3 K_{2}}{2} .
$$

Using (2.6) and (3.9) it is clear that for $\Phi_{0} \in E_{3 / 4,1 / 2}$, the solution exists globally in $E_{3 / 4,1 / 2}$, and since $E_{3 / 4,1 / 2} \subset E_{\xi, \xi-1 / 4}$, such a solution exists globally in $E_{\xi, \xi-1 / 4}$. Using the fact that $E_{3 / 4,1 / 2}$ is dense in $E_{\xi, \xi-1 / 4}$, we find that for $\xi \in[1 / 2,3 / 4]$ and $\Phi_{0} \in E_{\xi, \xi-1 / 4}$, the unique solution (2.3) of (2.2) exists globally in $E_{\xi, \xi-1 / 4}$.

For $R>0$ and $\Phi_{0} \in E_{\xi, \xi-1 / 4}$ such that $\left\|\Phi_{0}\right\|_{E_{\xi, \xi-1 / 4}}^{2} \leq R$, using (3.9), it follows that

$$
\left\|S_{\xi}(t) \Phi_{0}\right\|_{E_{\xi, \xi-1 / 4}}^{2} \leq 2 k_{9} / k_{7}, \quad \text { for all } t \geq t_{1},
$$

where

$$
t_{1}=t_{1}(R)=\frac{1}{\varepsilon} \ln \left[\left(k_{8} R+\|f\|_{0}^{2} / 2 \varepsilon\right) / k_{9}\right]
$$


Thus, the solution semiflow $S(t)$ generated by the solutions of $(2.2)$ is bounded dissipative in $E_{\xi, \xi-1 / 4}$.

4. Global attractor. Here we present the main result of this work, where we prove the existence of a global attractor for the solution semiflow generated by the solutions of $(2.2)$ in $E_{\xi, \xi-1 / 4}$, for $\xi \in$ $[1 / 2,3 / 4]$. Indeed, we show that the solution semiflow is $\kappa$-contracting, then Lemma 3.2 and the classical theory of global attractors provide the result.

Lemma 4.1. In $E_{\xi, \xi-1 / 4}, \xi \in[1 / 2,3 / 4]$, the semiflow $S_{\xi}(t)$ generated by the solutions of (2.2) is uniformly $\kappa$-contracting.

Proof. For $\xi \in[1 / 2,3 / 4]$, let $D$ be a bounded set in

$$
E_{\xi, \xi-1 / 4}, \quad \Phi_{0 i} \in E_{3 / 4,1 / 2} \cap D, \quad i=1,2,
$$

and let

$$
S_{\xi}(t) \Phi_{0 i}=\Phi_{i}(t)=\left(u_{i}(t), u_{i t}(t)\right)^{T}, \quad i=1,2
$$

be the solutions of (2.2) given by (2.5). Let

$$
Y(t)=\Phi_{1}(t)-\Phi_{2}(t)=\left(y(t), y_{t}(t)\right)^{T},
$$

where $y(t)=u_{1}(t)-u_{2}(t)$. Recalling Remark 3.1 and equation (1.3), we obtain

$$
\begin{aligned}
\left(1+\beta A^{1 / 2}\right) y_{t t} & +\delta A^{1 / 2} y_{t}+\alpha A y \\
& =g\left(\left\|u_{2}\right\|_{\xi-1 / 4}^{2}\right) A^{1 / 2} u_{2}-g\left(\left\|u_{1}\right\|_{\xi-1 / 4}^{2}\right) A^{1 / 2} u_{1}
\end{aligned}
$$

For $t>0$, let us consider the inner product of the last equation with $A^{2 \xi-1}\left(y_{t}+\varepsilon y\right)$ in $H, \varepsilon>0$, we get

$$
\frac{d}{d t} \Xi_{\xi}(t)+\Gamma_{\xi}(t)=G_{\xi}\left(t ; u_{1}, u_{2}\right)
$$

where

$$
\begin{aligned}
\Xi_{\xi}(t)= & \frac{1}{2}\left\|y_{t}\right\|_{\xi-1 / 2}^{2}+\frac{\beta}{2}\left\|y_{t}\right\|_{\xi-1 / 4}^{2}+\frac{\alpha}{2}\|y\|_{\xi}^{2}+\frac{\varepsilon \delta}{2}\|y\|_{\xi-1 / 4}^{2} \\
& +\varepsilon\left\langle y_{t}, y\right\rangle_{\xi-1 / 2}+\varepsilon \beta\left\langle y_{t}, y\right\rangle_{\xi-1 / 4} \\
& \Gamma_{\xi}(t)=(\delta-\varepsilon \beta)\left\|y_{t}\right\|_{\xi-1 / 4}^{2}-\varepsilon\left\|y_{t}\right\|_{\xi-1 / 2}^{2}+\varepsilon \alpha\|y\|_{\xi}^{2},
\end{aligned}
$$


and

$$
\begin{aligned}
G_{\xi}\left(t ; u_{1}, u_{2}\right)= & \left\langle g\left(\left\|u_{2}\right\|_{\xi-1 / 4}^{2}\right) A^{1 / 2} u_{2}\right. \\
& \left.-g\left(\left\|u_{1}\right\|_{\xi-1 / 4}^{2}\right) A^{1 / 2} u_{1}, A^{2 \xi-1}\left(y_{t}+\varepsilon y\right)\right\rangle_{0} .
\end{aligned}
$$

Recalling (1.7) and (3.4) it follows that, for sufficiently small $\varepsilon$,

$(4.3) \Xi_{\xi}(0) \leq \frac{1}{2}(1+\beta)(1+\varepsilon)\left\|y_{t}(0)\right\|_{\xi-1 / 4}^{2}+\frac{1}{2}(\alpha+\varepsilon(1+\beta+\delta))\|y(0)\|_{\xi}^{2}$,

(4.4) $\Xi_{\xi}(t) \geq \frac{1}{2}(\beta-\varepsilon(1+\beta))\left\|y_{t}(t)\right\|_{\xi-1 / 4}^{2}+\frac{1}{2}(\alpha-\varepsilon(1+\beta))\|y(t)\|_{\xi}^{2}$,

$$
\varepsilon \Xi_{\xi}(t)-\Gamma_{\xi}(t) \leq 0
$$

In such a case, using (4.2) and (4.5), we obtain

$$
\frac{d}{d t} \Xi_{\xi}(t)+\varepsilon \Xi_{\xi}(t) \leq G_{\xi}\left(t ; u_{1}, u_{2}\right), \quad \text { for all } t>0 \text {. }
$$

That is,

$$
\frac{d}{d t}\left(e^{\varepsilon t} \Xi_{\xi}(t)\right) \leq e^{\varepsilon t} G_{\xi}\left(t ; u_{1}, u_{2}\right), \quad \text { for all } t>0
$$

From (3.9), it follows that there exists $k_{10}=k_{10}(D)$ such that

$$
\left\|S_{\xi}(t) \Phi_{0 i}\right\|_{E_{\xi, \xi-1 / 4}}=\left(\left\|u_{i}(t)\right\|_{\xi}^{2}+\left\|u_{i t}(t)\right\|_{\xi-1 / 4}^{2}\right)^{1 / 2} \leq k_{10},
$$

for all $t \geq 0, i=1,2$. Taking into account (1.7), (2.1) and (4.7), there exists $k_{11}=k_{11}(D)$ such that for $t \geq 0$,

$$
\begin{aligned}
G_{\xi}\left(t ; u_{1}, u_{2}\right)= & \left\langle\left(g\left(\left\|u_{2}\right\|_{\xi-1 / 4}^{2}\right)-g\left(\left\|u_{1}\right\|_{\xi-1 / 4}^{2}\right)\right) A^{1 / 2} u_{1}\right. \\
& \left.-g\left(\left\|u_{2}\right\|_{\xi-1 / 4}^{2}\right) A^{1 / 2} y, A^{2 \xi-1}\left(y_{t}+\varepsilon y\right)\right\rangle_{0} . \\
= & \left(g\left(\left\|u_{2}\right\|_{\xi-1 / 4}^{2}\right)-g\left(\left\|u_{1}\right\|_{\xi-1 / 4}^{2}\right)\left\langle u_{1}, y_{t}+\varepsilon y\right\rangle_{\xi-1 / 4}\right. \\
& -g\left(\left\|u_{2}\right\|_{\xi-1 / 4}^{2}\right)\left\langle y, y_{t}+\varepsilon y\right\rangle_{\xi-1 / 4} \\
\leq & \left|g\left(\left\|u_{2}\right\|_{\xi-1 / 4}^{2}\right)-g\left(\left\|u_{1}\right\|_{\xi-1 / 4}^{2}\right)\right|\left\|u_{1}\right\|_{\xi-1 / 4}\left\|y_{t}+\varepsilon y\right\|_{\xi-1 / 4} \\
& +\left|g\left(\left\|u_{2}\right\|_{\xi-1 / 4}^{2}\right)\right|\|y\|_{\xi-1 / 4}\left\|y_{t}+\varepsilon y\right\|_{\xi-1 / 4} \leq k_{11}\|y\|_{\xi-1 / 4}
\end{aligned}
$$


Substituting the previous inequality into (4.6) and integrating the result from 0 into $t>0$, we get

$$
\Xi_{\xi}(t) \leq e^{-\varepsilon t} \Xi_{\xi}(0)+\varepsilon^{-1} k_{11} \max _{l \in[0, t]}\|y(l)\|_{\xi-1 / 4}
$$

We can choose $\varepsilon>0$ sufficiently small such that

$$
\min \{\beta-\varepsilon(1+\beta) \alpha-\varepsilon(1+\beta)\}>0
$$

Let

$$
\begin{aligned}
& k_{12}=\frac{1}{2} \max \{(1+\beta)(1+\varepsilon), \alpha+\varepsilon(1+\beta+\delta)\}, \\
& k_{13}=\frac{1}{2} \min \{(\beta-\varepsilon(1+\beta)),(\alpha-\varepsilon(1+\beta))\}>0 .
\end{aligned}
$$

In such a case, recalling (4.3) and (4.4), we find

(4.9) $\Xi_{\xi}(0) \leq k_{12}\left(\left\|y_{t}(0)\right\|_{\xi-1 / 4}^{2}+\|y(0)\|_{\xi}^{2}\right)=k_{12}\left\|\Phi_{01}-\Phi_{02}\right\|_{E_{\xi, \xi-1 / 4}}^{2}$,

$k_{13}\left\|S_{\xi}(t) \Phi_{01}-S_{\xi}(t) \Phi_{02}\right\|_{E_{\xi, \xi-1 / 4}}^{2}=k_{13}\left(\left\|y_{t}(t)\right\|_{\xi-1 / 4}^{2}+\|y(t)\|_{\xi}^{2}\right)^{1 / 2} \leq \Xi_{\xi}(t)$.

Using (4.8)-(4.10), it follows that

$$
\begin{aligned}
\| S_{\xi}(t) \Phi_{01} & -S_{\xi}(t) \Phi_{02}\left\|_{E_{\xi, \xi-1 / 4}}^{2} \leq \frac{k_{12}}{k_{13}} e^{-\varepsilon t}\right\| \Phi_{01}-\Phi_{02} \|_{E_{\xi, \xi-1 / 4}}^{2} \\
+ & \frac{k_{11}}{\varepsilon k_{13}} \max _{l \in[0, t]}\left\|u_{1}(l)-u_{2}(l)\right\|_{\xi-1 / 4}, \quad \text { for all } t>0,
\end{aligned}
$$

that is,

$$
\begin{aligned}
\| S_{\xi}(t) \Phi_{01} & -S_{\xi}(t) \Phi_{02}\left\|_{E_{\xi, \xi-1 / 4}} \leq\left(\frac{k_{12}}{k_{13}}\right)^{1 / 2} e^{-\varepsilon t / 2}\right\| \Phi_{01}-\Phi_{02} \|_{E_{\xi, \xi-1 / 4}} \\
& +\left(\frac{k_{11}}{\varepsilon k_{13}}\right)^{1 / 2} \max _{l \in[0, t]}\left\|u_{1}(l)-u_{2}(l)\right\|_{\xi-1 / 4}^{1 / 2}, \quad \text { for all } t>0 .
\end{aligned}
$$

Since $E_{3 / 4,1 / 2}$ is dense in $E_{3 / 4,1 / 2}$, one can see that the above inequality is satisfied for $\Phi_{0 i} \in D, i=1,2$. 
Now we shall apply the Ascoli-Arzela theorem in order to prove that for all $t>0$,

$$
\rho_{t}\left(\Phi_{01}, \Phi_{02}\right)=\left(\frac{k_{11}}{\varepsilon k_{13}}\right)^{1 / 2} \max _{l \in[0, t]}\left\|u_{1}(l)-u_{2}(l)\right\|_{\xi-1 / 4}^{1 / 2},
$$

is a precompact pseudometric in $E_{\xi, \xi-1 / 4}$. Indeed, given a bounded sequence $\left\{\Phi_{0}^{n}\right\}_{n=1}^{\infty}$ in $E_{\xi, \xi-1 / 4}$, let $S_{\xi}(t) \Phi_{0}^{n}=\Phi_{n}(t)=\left(u_{n}(t), u_{n t}(t)\right)^{T}, n=$ $1,2, \ldots$, be the solutions of (2.2). From (3.9), it is clear that there exists a constant $k_{14}=k_{14}\left(\left\{\Phi_{0}^{n}\right\}\right)>0$ such that

$$
\left\|S_{\xi}(t) \Phi_{0}^{n}\right\|_{E_{\xi, \xi-1 / 4}}^{2}=\left\|u_{n}(t)\right\|_{\xi}^{2}+\left\|u_{n t}(t)\right\|_{\xi-1 / 4}^{2} \leq k_{14}^{2},
$$

for all $t \geq 0, n=1,2, \ldots$ Using (4.12) and the fact that $V^{\xi}$ is compactly embedded in $V^{\xi-1 / 4}$, we find that $\left\{u_{n}(t)\right\}_{n=1}^{\infty}$ is precompact in $V^{\xi-1 / 4}$, for all $t \geq 0$. From the Sobolev embedding theorem, we know that $V^{\xi-1 / 4}$ is compactly embedded in

$$
C_{B}(0,1)=\{f: f \text { is a continuous and bounded function on }(0,1)\},
$$

that is, there exists a constant $k_{15}$ such that

$$
\sup _{\rho \in(0,1)}|\varphi(\rho)| \leq k_{15}\|\varphi\|_{\xi-1 / 4}^{2}, \quad \text { for all } \varphi \in V^{\xi-1 / 4} \text {. }
$$

From (4.12) and the last inequality, it follows that

$$
\sup _{\rho \in(0,1)}\left|u_{n t}(\rho, t)\right| \leq k_{16}, \quad \text { for all } t \geq 0, n=1,2, \ldots,
$$

where $k_{16}=k_{14} k_{15}$. In such a case, using the mean value theorem, we find

$$
\begin{array}{r}
\left\|u_{n}\left(t_{1}\right)-u_{n}\left(t_{2}\right)\right\|_{\xi-1 / 4} \leq k_{16}\left|t_{1}-t_{2}\right|, \\
\text { for all } t_{1}, t_{2} \geq 0, n=1,2, \ldots,
\end{array}
$$

that is, the sequence $\left\{u_{n}(t)\right\}_{n=1}^{\infty}$, as $V^{\xi-1 / 4}$-valued functions, is equicontinuous in $t$, for all $t \geq 0$. In such a case, using the Ascoli-Arzela theorem, there is a subsequence of $\left\{u_{n}(t)\right\}_{n=1}^{\infty}$ which is convergent in the Frechet space $L_{\mathrm{loc}}^{\infty}\left[0, \infty ; V^{\xi-1 / 4}\right)$ and this is a Cauchy sequence with respect to $\rho_{t}\left(\Phi_{01}, \Phi_{02}\right)$. Thus $\rho_{t}$ is a precompact pseudometric on $E_{\xi, \xi-1 / 4}$.

Taking into account $(4.11)$, since $\Psi(t)=\left(k_{12} / k_{13}\right)^{1 / 2} e^{-\varepsilon t / 2}$ is a nonincreasing function such that $\Psi(t) \rightarrow 0$ as $t \rightarrow \infty$ and we proved 
that $\rho_{t}$ is a precompact pseudometric on $E_{\xi, \xi-1 / 4}$. From [13, Lemma 22.5] we find that the semigroup $S_{\xi}(t)$ on $E_{\xi, \xi-1 / 4}$ is uniformly $\kappa$ contracting.

Now we are ready to introduce the main result of this work.

Theorem 4.2. Given $\xi \in[1 / 2,3 / 4]$, there exists a global attractor $\mathcal{A}_{\xi, \xi-1 / 4}$ for the solution semiflow $S_{\xi}(t)$ generated by the solutions of $(2.2)$ in $E_{\xi, \xi-1 / 4}$ which attracts every bounded set in $E_{\xi, \xi-1 / 4}$.

Proof. From Lemmas 3.2 and 4.1, we know that for $\xi \in[1 / 2,3 / 4]$, the semiflow $S_{\xi}(t)$ is bounded dissipative and $\kappa$-contracting in $E_{\xi, \xi-1 / 4}$. In such a case, following the classical theory of global attractors we get the result.

\section{REFERENCES}

1. J.M. Ball, Initial-boundary value problems for an extensible beam, J. Math. Anal. Appl. 41 (1973), 61-90.

2. M. Coti Zelati, Global and exponential attractors for the singularly perturbed extensible beam, Discr. Cont. Dynam. Syst. 25 (2009), 1041-1060.

3. H. Gao, Global dynamics of a nonlinear beam equation with strong structural damping, J. Partial Diff. Eqs. 12 (1999), 324-336.

4. J.M. Ghidaglia and A. Marzocchi, Long time behavior of strongly damped wave equations, global attractors and their dimensions, SIAM J. Math. Anal. 22 (1991), 879-895.

5. C. Giorgi, M.G. Naso, V. Pata and M. Potomkin, Global attractors for the extensible thermoelastic beam system, J. Diff. Eqs. 9 (2009), 3496-3517.

6. C. Giorgi, V. Pata and E. Vuk, On the extensible viscoelastic beam, Nonlinearity 21 (2008), 713-733.

7. M. Grasselli, V. Pata and G. Prouse, Longtime behavior of a viscoelastic Timoshenko beam, Discr. Cont. Dynam. Syst. 10 (2004), 337-348.

8. J. Hale, Asymptotic behavior of dissipative systems, Math. Surv. Mono. 25, American Mathematical Society, Providence, RI, 1988.

9. T.F. Ma and V. Narciso, Global attractor for a model of extensible beam with nonlinear damping and source terms, Nonlin. Anal. 73 (2010), 3402-3412.

10. A. Pazy, Semigroups of linear operators and applications to partial differential equations, Appl. Math. Sci. 44, Springer-Verlag, New York, 1983.

11. G. Perla Menzala and E. Zuazua, Timoshenko's beam equation as limit of a nonlinear one-dimensional von Kármán system, Proc. Roy. Soc. Edinb. 130 (2000), $855-875$. 
12. R. Racke and C. Shang, Global attractors for nonlinear beam equations, Proc. Roy. Soc. Edinb. 142 (2012), 1087-1107.

13. G.R. Sell and Y. You, Dynamics of evolutionary equations, Appl. Math. Sci. 143, Springer-Verlag, New York, 2002.

14. D. Sevcovic, Existence and limiting behavior for damped nonlinear evolution equations with nonlocal terms, Comm. Math. Univ. Carolin. 31 (1990), 283-293.

15. M. Tucsnak, Semi-internal stabilization for a nonlinear Bernoulli-Euler equation, Math. Meth. Appl. Sci. 19 (1996), 897-907.

Department of Mathematics, The University of Jordan, Amman 11942, JORDAN

Email address: axfarah@gmail.com 